\title{
Food Sublingual Immunotherapy Using Consistent, Cheaper and Customizable Oral Immunotherapy Solutions
}

\section{Karna Gendo \\ Tony Orden \\ Allyson Tevrizian \\ Joshua Jacobs \\ Nancy Mozelsio \\ Katherine Gilbert \\ Matthew Lodewick}

Allergy and Asthma Medical Group of the Bay Area, Walnut Creek, CA, 94598, USA
Correspondence: Karna Gendo

Allergy and Asthma Medical Group of the Bay Area, Walnut Creek, CA, 94598, USA

Tel +I 925 935-6252

$\mathrm{Fax}+1925930-0942$

Email kbgendo@mac.com

\begin{abstract}
Food SLIT (sublingual immunotherapy) is a food desensitization method with a daily maintenance dose of $<10 \mathrm{mg}$ for up to 5 years. Many protocols use commercially available skin test extracts. Oral immunotherapy (OIT) treatment solutions have consistent protein concentrations, are comparatively cheaper and customizable and therefore were adapted for use in a published SLIT dosing schedule.
\end{abstract}

Keywords: food allergy, immunotherapy, pharmacoeconomics, food allergy treatments, sublingual immunotherapy, desensitization

Food Sublingual Immunotherapy (SLIT) is a food desensitization method with a typical daily maintenance dose of $<10 \mathrm{mg}$ protein for up to 3-5 years, which has been shown to increase tolerance in hazelnut and peanut allergic patients in randomized, double-blind, placebo-controlled trials. ${ }^{1,2}$ In SLIT, intact food protein is given sublingually to the tolerogenic antigen-presenting Langerhans cells, held for 2 minutes, and then swallowed or spat out. Starting doses are at the microgram level and increase to milligram doses by maintenance. ${ }^{3}$ SLIT buffers against unintentional food exposure while maintaining a favorable safety profile with mostly transient oropharyngeal itching in less than $5 \%$ of peanut SLIT doses. ${ }^{4}$ Many published SLIT protocols use commercially available skin test extracts.

Skin test extracts have relatively higher protein concentration variability and cost, and lower customizability in comparison to Oral Immunotherapy (OIT) solutions. Egg and milk skin test extracts contain 2-4 mg protein per milliliter, while peanut extract contains $4-8 \mathrm{mg}$ protein per milliliter at an approximate price of 4 US dollars per mg protein. ${ }^{5}$ Allergists collaborate to develop and refine OIT protocols which yield solutions with reasonable protein concentration consistency. ${ }^{6,7}$ Since actual food is used to make OIT solutions, the cost is orders of magnitude less. Although OIT solutions have a shorter shelf life than skin test extracts, they can be frozen for future use. ${ }^{8}$ An OIT solution can be made to approximate the protein concentration of a skin test extract and substituted for the skin test extract used in a published SLIT protocol ${ }^{4}$ (Figure 1) and is found to dispense easily (Figure 2). If for any reason, the maintenance dose needs to be adjusted, the OIT solution can be customized without changing the protocol, which is difficult to do with skin test extracts which have variable, fixed protein concentrations (Table 1). 
Dilution $1-1: 1$

- To start out you will be taking one aliquot of food plus flavoring and adding distilled water $(<25$ $\mathrm{ml}$ ) up to a final volume of $25 \mathrm{ml}$. This will be your Food SLIT Dilution 1, which you will use to make Dilutions 2, 3, and 4.

- To make vials for a 3-month supply renewal: you will be taking 2 aliquots of food and adding distilled water $(<50 \mathrm{ml})$ up to a final volume of $50 \mathrm{ml}$. Shelf life is 2 weeks unless frozen.

- Patients will advance every 14 days and begin with 1 drop $(0.05 \mathrm{ml} \times 14)$, followed by 2 drops $(0.10 \mathrm{ml} \mathrm{x14})$, followed by 4 drops $(0.20 \mathrm{ml} \times 14)$, and finishing with 8 drops for 14 days $(0.40 \mathrm{ml}$ $\mathrm{x} 14)$. This totals $10.50 \mathrm{ml}$ needed.

- Then patients will be maintaining 8 drops daily which equals $0.40 \mathrm{ml}$, requiring $12 \mathrm{ml}$ per month. $(0.40 \mathrm{ml} \times 30$ days $=12 \mathrm{ml} / \mathrm{month})$

Dilution 2 - 1:10

- Take $1.5 \mathrm{ml}$ from Dilution 1 (Stock) and place into $13.5 \mathrm{ml}$ distilled water. This is now dilution 2.

- Patients will advance every 14 days and begin with 1 drop $(0.05 \mathrm{ml} \times 14)$, followed by 2 drops $(0.10 \mathrm{ml} \mathrm{x14})$, followed by 4 drops $(0.20 \mathrm{ml} \times 14)$, and finishing with 8 drops for 14 days $(0.40 \mathrm{ml}$ $\mathrm{x} 14)$. This totals $10.50 \mathrm{ml}$ needed.

Dilution 3 - 1:100

- Take $1.5 \mathrm{ml}$ from Dilution 2 and place into $13.5 \mathrm{ml}$ distilled water. This is now dilution 3.

- Patients will advance every 14 days and begin with 1 drop $(0.05 \mathrm{ml} \times 14)$, followed by 2 drops $(0.10 \mathrm{ml} \times 14)$, followed by 4 drops $(0.20 \mathrm{ml} \times 14)$, and finishing with 8 drops for 14 days $(0.40 \mathrm{ml}$ $\mathrm{x} 14)$. This totals $10.50 \mathrm{ml}$ needed.

Dilution 4 - 1:1,000

- Take $0.5 \mathrm{ml}$ from Dilution 3 and place into $4.5 \mathrm{ml}$ distilled water. This is now dilution 4 .

- Patients will advance every 14 days and begin with 1 drop $(0.05 \mathrm{ml} \times 14$ and finish with 5 drops $(0.25 \mathrm{ml} \times 14)$. This totals $4.2 \mathrm{ml}$ needed.

Figure I Food SLIT dilution protocol.

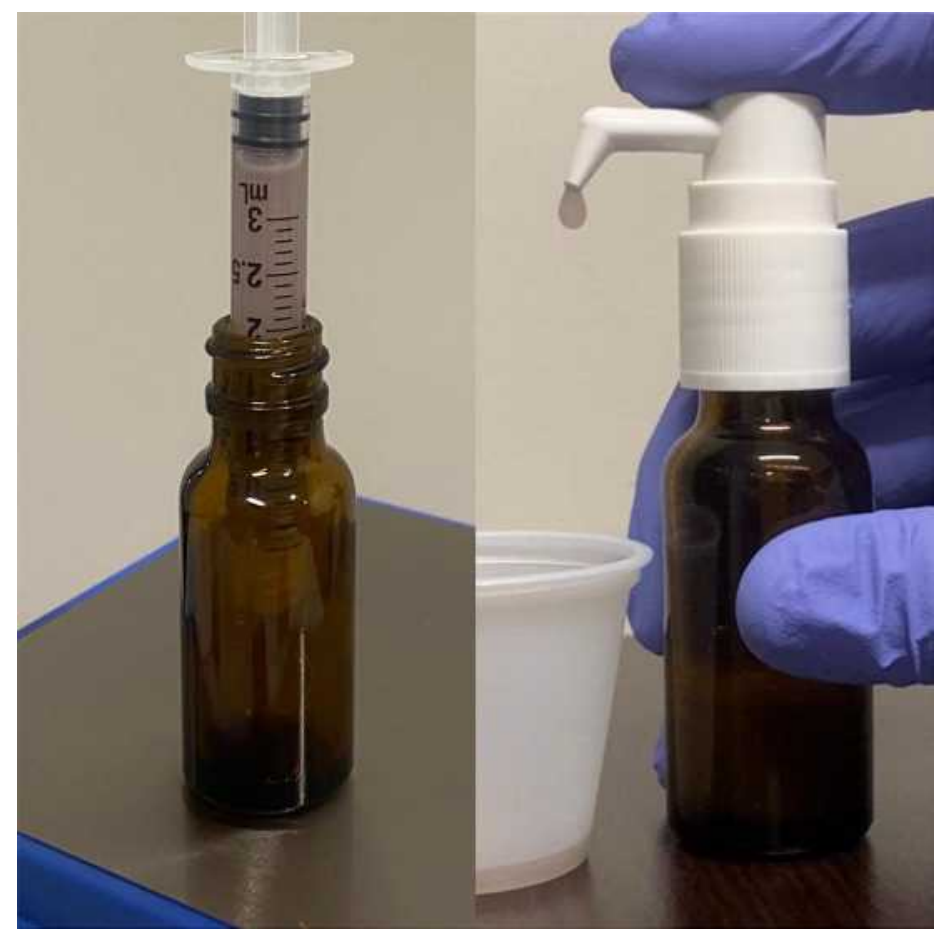

Figure 2 Peanut dilution I - I:I $5 \mathrm{mg} / \mathrm{mL}$ plus flavoring. 
Table I Summary of Food Amounts Needed to Create a SLIT Maintenance Vial Using an OIT Dosing Protocol

\begin{tabular}{|c|c|c|c|c|}
\hline Food & $\begin{array}{l}\text { Maintenance } \\
\text { Dose (mg } \\
\text { Protein) }\end{array}$ & $\begin{array}{l}\text { Aliquot of Food } \\
\text { in } 25 \mathrm{~mL} \text { Vial }\end{array}$ & Flavoring & Notes \\
\hline Almond & $2 \mathrm{mg}$ & $\begin{array}{l}\text { I/8 tsp almond } \\
\text { butter }\end{array}$ & Kool-Aid 0.5 tsp & Barney Butter Almond Butter - Smooth \\
\hline Cashew & $2 \mathrm{mg}$ & $\begin{array}{l}\text { I/8 tsp cashew } \\
\text { butter }\end{array}$ & Kool-Aid 0.5 tsp & Artisana Raw Organic Cashew Butter \\
\hline Chickpea & $2 \mathrm{mg}$ & $625 \mathrm{mg}$ flour & Kool-Aid 0.5 tsp & $\begin{array}{l}\text { Garbanzo Bean Flour Bob's Red Mill } \\
\text { www.bobsredmill.com }\end{array}$ \\
\hline Coconut & $2 \mathrm{mg}$ & $\begin{array}{l}3 / 8 \text { tsp or } 875 \mathrm{mg} \\
\text { flour }\end{array}$ & Kool-Aid 0.5 tsp & $\begin{array}{l}\text { Bob's Red Mill Organic Coconut Flour } \\
\text { http://www.bobsredmill.com/organic-coconut- } \\
\text { flour.html }\end{array}$ \\
\hline $\begin{array}{l}\text { Egg } \\
\text { White }\end{array}$ & $2 \mathrm{mg}$ & I.I $\mathrm{mL}$ egg white & Kool-Aid 0.5 tsp & $\begin{array}{l}\text { All Whites } 100 \% \text { or Eggland's Best Liquid Egg } \\
\text { Whites }\end{array}$ \\
\hline Hazelnut & $2 \mathrm{mg}$ & $\begin{array}{l}\text { I5 mL milk or } \\
875 \mathrm{mg} \text { flour }\end{array}$ & $\begin{array}{l}\text { Kool-Aid } 0.5 \text { tsp for flour, Chocolate } \\
\text { or strawberry syrup for milk }\end{array}$ & $\begin{array}{l}\text { Elmhurst Hazelnut Milk or Bob's Red Mill } \\
\text { www.bobsredmill.com/hazelnut-flour-meal.html }\end{array}$ \\
\hline Milk & $2 \mathrm{mg}$ & $3.75 \mathrm{~mL}$ milk & Chocolate or strawberry syrup & $\begin{array}{l}\text { Use milk > } 10 \text { days from expiration date. Use } \\
\text { organic or irradiated whole milk }\end{array}$ \\
\hline Milk & $7 \mathrm{mg}$ & I3.I mL milk & Chocolate or strawberry syrup & $\begin{array}{l}\text { Use milk > } 10 \text { days from expiration date. Use } \\
\text { organic or irradiated whole milk }\end{array}$ \\
\hline Peanut & $2 \mathrm{mg}$ & $\begin{array}{l}\text { I/8 tsp peanut } \\
\text { butter }\end{array}$ & Kool-Aid 0.5 tsp & $\begin{array}{l}\text { Peanut Butter \& Company, Santa Cruz Organic } \\
\text { or Jif peanut butters. Stir for } 2 \text { hours }\end{array}$ \\
\hline Rye & $2 \mathrm{mg}$ & $938 \mathrm{mg}$ flour & Kool-Aid 0.5 tsp & Organic Dark Rye Flour at www.nuts.com \\
\hline Sesame & $1.7 \mathrm{mg}$ & I/8 tsp tahini & Kool-Aid 0.5 tsp & Max Sesame Tahini Spread \\
\hline Shrimp & & $\begin{array}{l}\text { Consider dust } \\
\text { mite SLIT }\end{array}$ & & $\begin{array}{l}\text { Eur Ann Allergy Clin Immunol. 20II } \\
\text { Oct;43(5):162-4 }\end{array}$ \\
\hline Soy & $2 \mathrm{mg}$ & $3.75 \mathrm{~mL}$ soy milk & Chocolate or strawberry syrup & $\begin{array}{l}\text { Use milk > } 10 \text { days from expiration date. Use } \\
\text { Original Silk Soy Milk }\end{array}$ \\
\hline Sunflower & $2 \mathrm{mg}$ & $567 \mathrm{mg}$ butter & Kool-Aid 0.5 tsp & Sunbutter \\
\hline Walnut & $1.7 \mathrm{mg}$ & $\begin{array}{l}\text { I/8 tsp walnut } \\
\text { butter }\end{array}$ & Kool-Aid 0.5 tsp & www.fastachi.com Raw Walnut Butter \\
\hline Wheat & $2 \mathrm{mg}$ & $178 \mathrm{mg}$ flour & Kool-Aid 0.5 tsp & Druids Grove Vital Wheat Gluten Flour \\
\hline
\end{tabular}

We hope that this adaption of OIT solutions for use in available SLIT protocols improves the access to consistent, economical, and customizable food desensitization.

\section{Disclosure}

Dr Joshua Jacobs reports consultancy fees and contracted research from Aimmune, outside the submitted work. The authors report no other conflicts of interest in this work.

\section{References}

1. Enrique E, Pineda F, Malek T, et al. Sublingual immunotherapy for hazelnut food allergy: a randomized, double-blind, placebo-controlled study with a standardized hazelnut extract. J Allergy Clin Immunol. 2005;116(5):1073-1079. doi:10.1016/j.jaci.2005.08.027

2. Fleischer DM, Burks AW, Vickery BP, et al.; Consortium of Food Allergy Research (CoFAR). Sublingual immunotherapy for peanut allergy: a randomized, double-blind, placebo-controlled multicenter trial. J Allergy Clin Immunol. 2013;131(1):119-127. doi:10.1016/j. jaci.2012.11.011 
3. McGowan EC, Wood RA. Sublingual (SLIT) versus oral immunotherapy (OIT) for food allergy. Curr Allergy Asthma Rep. 2014;14(12):486. doi:10.1007/s11882-014-0486-9

4. Kim EH, Yang L, Ye P, et al. Long-term sublingual immunotherapy for peanut allergy in children: clinical and immunologic evidence of desensitization. J Allergy Clin Immunol. 2019;144(5):1320-1326. doi:10.1016/j.jaci.2019.07.030

5. Communication with N. Isenhour, Sept 12, 2019, Stallergenes Greer Inc. Ingestant egg, milk, peanut extracts. 55 Cambridge Parkway, Suite 400, Cambridge, MA 02142.
6. Filep S, Block DS, Smith BR, et al. Specific allergen profiles of peanut foods and diagnostic or therapeutic allergenic products. $J$ Allergy Clin Immunol. 2018;141(2):626-631. doi:10.1016/j. jaci.2017.05.049

7. Wasserman R. OIT packet nuts and other foods; North Texas. Available from: www.allergypartners.com. Accessed April 14, 2021.

8. Sagara N, Fujita S, Suzuki R, et al. Successful sublingual immunotherapy for severe egg allergy in children: a case report. Allergy Asthma Clin Immunol. 2021;17(1):1-4. doi:10.1186/s13223-02000506

\section{Publish your work in this journal}

The Journal of Asthma and Allergy is an international, peer-reviewed open-access journal publishing original research, reports, editorials and commentaries on the following topics: Asthma; Pulmonary physiology; Asthma related clinical health; Clinical immunology and the immunological basis of disease; Pharmacological interventions and

Submit your manuscript here: https://www.dovepress.com/journal-of-asthma-and-allergy-journal new therapies. The manuscript management system is completely online and includes a very quick and fair peer-review system, which is all easy to use. Visit http://www.dovepress.com/testimonials.php to read real quotes from published authors. 